\title{
Benign Metastasizing Leiomyoma Presenting as Cavitating Lung Nodules
}

\author{
Angeliki A Loukeri MD, Ioannis N Pantazopoulos MD MSc, Rodoula Tringidou MD PhD, \\ Pantelis Giampoudakis MD, Argyro Valaskatzi MD, Pinelopi A Loukeri MD, and \\ Christos F Kampolis MD
}

\begin{abstract}
Benign metastasizing leiomyoma (BML) was initially used to describe single or multiple pulmonary nodules composed of proliferating smooth muscle cells (lacking cellular atypia) in premenopausal females 3 months to $20 \mathrm{y}$ after hysterectomy for uterine leiomyoma. The lung is the most commonly involved site, thus including many malignant and benign entities in the differential diagnosis. The present case refers to a 47-y-old premenopausal woman with a history of subtotal hysterectomy for a uterine leiomyoma presenting with bilateral cavitating pulmonary nodules. A number of nodules were resected by video-assisted thoracoscopic surgery. The histological findings in correlation with the immunohistochemical results were consistent with the diagnosis of BML. A bilateral salpingooophorectomy was performed, combined with complete removal of the remaining cervix. One year later, the subject remains asymptomatic, and the pulmonary nodules are stable with regard to number, size, location, and morphology. Key words: pulmonary nodules; cavitary; uterine leiomyoma; benign; hysterectomy; premenopause. [Respir Care 2014;59(7):e94-e97. (C) 2014 Daedalus Enterprises]
\end{abstract}

\section{Introduction}

Benign metastasizing leiomyoma (BML) is a rare entity that usually presents in women who have previously undergone hysterectomy for uterine leiomyomatosis. The typical clinical presentation consists of multiple, benign, slowgrowing, smooth muscle tumors in various locations outside the uterus. Lungs are the most frequent site of involvement, whereas the abdomen, skin, mediastinum, nervous system, and bones are less commonly affected. Pulmonary nodules of variable sizes that rarely cavitate are the most

Dr Loukeri is affiliation with the Respiratory Intensive Care Unit; Drs Pantazopoulos, Giampoudakis, and Kampolis are affiliated with the 12th Respiratory Medicine Department; and Drs Tringidou and Valaskatzi are affiliated with the Pathology Department, Athens Chest Hospital "Sotiria," Athens, Greece. Dr Loukeri is affiliated with the 1st Paediatric Clinic, "Aghia Sophia" Children's Hospital, National and Kapodistrian University of Athens Medical School, Athens, Greece.

The authors have disclosed no conflicts of interest.

Correspondence: Angeliki A Loukeri MD, Respiratory Intensive Care Unit, Athens Chest Hospital "Sotiria," Mesogeion 152, 11527 Athens, Greece. E-mail: angelouk@live.com.

DOI: $10.4187 /$ respcare. 02775 common radiological finding. We report the case of a 47-y-old woman with BML presenting with cavitating pulmonary nodules.

\section{Case Report}

A 47-y-old premenopausal woman (current smoker, 50 pack-years) was referred to our department for evaluation of nonproductive cough lasting several days. On admission, she had no additional respiratory or systemic symptoms, such as dyspnea, fever, hemoptysis, pleuritic chest pain, weight loss, or fatigue. Her medical history included a subtotal hysterectomy 9 y previous for a uterine leiomyoma, a colonoscopic polypectomy 2 months before admission, and surgical correction of a rectovaginal fistula in childhood. She had no family history of gynecological malignancy, had two normal deliveries, and had never received oral contraceptives.

Apart from a hysterectomy scar over the lower abdomen, physical examination on admission was unremarkable. Chest x-ray showed multiple bilateral nodular opacities (Fig. 1). Laboratory studies, including arterial blood gas analysis, complete blood count, and erythrocyte sedimentation rate, were within normal ranges. Skin testing for tuberculosis was reactive with a $25-\mathrm{mm}$ diameter of indu- 


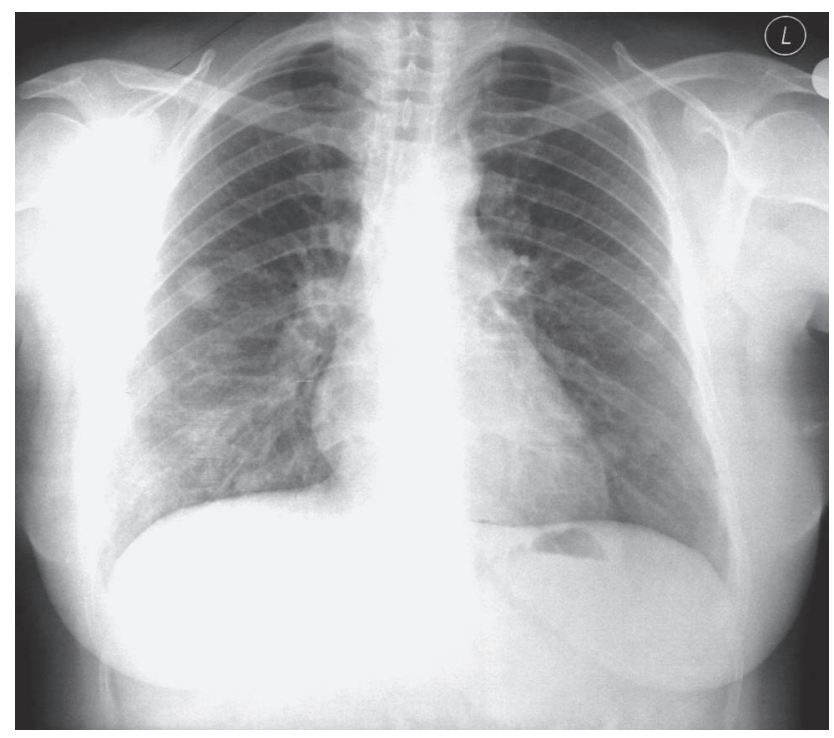

Fig. 1. Posteroanterior radiograph of chest shows multiple bilateral nodules.
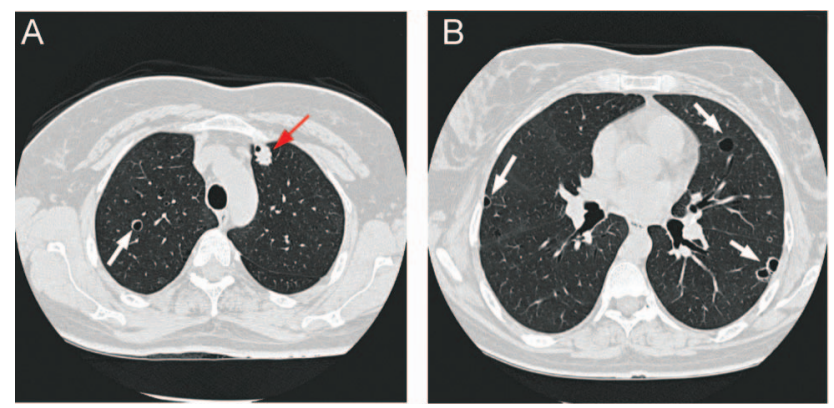

Fig. 2. Initial computed tomography scan shows a partially cavitated nodule (red arrow) and thin-walled cavities (white arrows) in the upper and lower lung fields bilaterally.

ration. Sputum cytology, culture, and stains for common bacteria and mycobacteria were negative. Blood tests for collagen vascular disease (anti-neutrophil antibody and cytoplasmic and perinuclear anti-neutrophil cytoplasmic antibodies) and serologic testing for viral or microbial infections were negative as well. Tumor markers (including cancer antigen 125, cancer antigen 19-9, and carcinoembryonic antigen) were all negative.

A computed tomography (CT) scan confirmed the presence of multiple well-circumscribed nodules of varying sizes, some with central cavitation, throughout both lung fields (Fig. 2), without involvement of the mediastinum or pleural space. Because of the presence of bilateral lung nodules, a common appearance of metastatic lesions, a search for a primary carcinoma was performed. Mammography and thyroid ultrasound excluded breast or thyroid neoplasia. An abdominal and pelvic CT scan revealed a cyst in the right ovary and cystic formation in the liver.
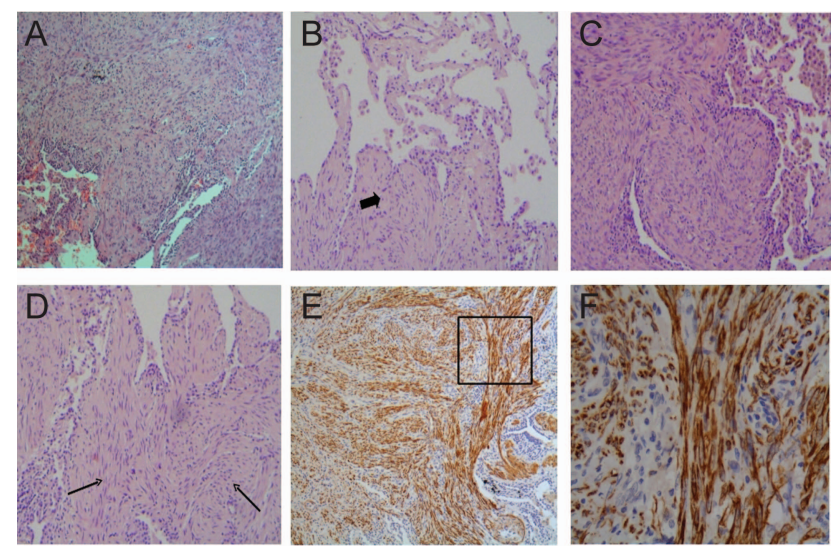

Fig. 3. Histological features of benign metastasizing leiomyoma $(B M L)$ in the lung. A: Area of BML in the lung parenchyma (hematoxylin and eosin stain, $\times 40$ ). B: Area of BML in a partially cavitated nodule of the left upper lobe (arrow; hematoxylin and eosin stain, $\times 100)$. C: Area of BML in a solid nodule of the left lower lobe (hematoxylin and eosin stain, $\times 100$ ). D: Area with spindle and round neoplastic cells (arrows; hematoxylin and eosin stain, $\times 100)$. E: Smooth muscle actin (SMA) positivity in spindle and round neoplastic cells in BML (SMA stain, $\times 100)$. F: Higher magnification of the square area in panel E of SMA positivity (SMA stain, $\times 400)$.

Fiberoptic bronchoscopy was carried out, and no endobronchial lesions were observed. Cytological analysis, Gram and acid-fast stains, and cultures for common bacteria and mycobacteria of bronchoalveolar lavage fluid were negative. Endobronchial biopsies from normal-appearing mucosa did not reveal neoplasia or inflammatory disease. Subsequent percutaneous fine-needle biopsy of the lesions was not diagnostic, so a number of nodules were resected by video-assisted thoracoscopic surgery. Microscopic examination of all surgical specimens showed multiple nodules of various sizes $(1-3 \mathrm{~cm}$ in diameter) with features of mesenchymal desmoplastic neoplasm. The histological findings in correlation with the immunohistochemical results (vimentin $[+]$, smooth muscle actin $[+]$, desmin $[+]$, estrogen receptor $[+], S-100$ protein $[-]$, HMB45 antigen $[-]$, and Ki-67 antigen $[+]<3 \%$ ) were consistent with the diagnosis of BML (Fig. 3).

$\left[{ }^{18} \mathrm{~F}\right]$ Fluorodeoxyglucose $\left({ }^{18} \mathrm{~F}-\mathrm{FDG}\right)$ positron emission tomography (PET)/CT was performed 1 month after surgery to evaluate the primary lesion and to identify potential metastases. The majority of multiple bilateral pulmonary nodules were not avid for 18F-FDG, with the exception of the larger one ( $2 \mathrm{~cm}$ in diameter) situated in the right upper lobe, which had mild 18F-FDG uptake (maximum standard uptake value of 2.2) (Fig. 4). Increased 18F-FDG uptake was also observed in the left lung at the site of surgical biopsy. There were no signs of distant malignancy.

Because metastatic lung lesions of BML may disappear following resection of the primary lesion, therefore indi- 

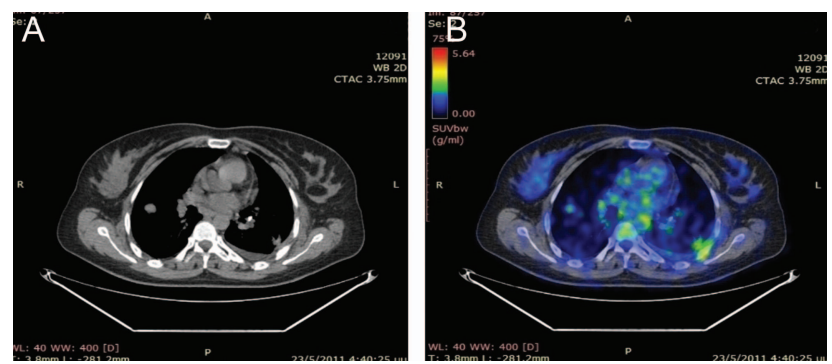

Fig. 4. The larger of multiple lung nodules $(2 \mathrm{~cm}$ in diameter), situated in the right upper lobe, had mild $\left[{ }^{18} \mathrm{~F}\right]$ fluorodeoxyglucose uptake (maximum standard uptake value of 2.2) in a PET/CT scan.

cating hormone dependence, the subject was referred to gynecologists for further evaluation and treatment planning. Bilateral salpingo-oophorectomy was performed, and the cervix remaining from the previous subtotal hysterectomy was removed with simultaneous dissection of both ureters. A thorough examination and evaluation of the whole abdomen revealed no further macroscopic disease, and peritoneal washings for cytological examination and an omental biopsy for pathologic analysis were obtained. Histological examination of all specimens did not reveal any evidence of disease. The postoperative period was uneventful. Pulmonary lesions remained stable in terms of number, size, location, and morphology in a CT scan performed 1 y later.

\section{Discussion}

Benign metastasizing leiomyoma is a term initially used by Steiner in 1939 to describe single or multiple pulmonary nodules composed of proliferating smooth muscle cells (lacking cellular atypia) in premenopausal females after hysterectomy for uterine leiomyoma. ${ }^{1}$ It is a rare condition (eg, less than 200 recorded before 2010) occurring 3 months to $20 \mathrm{y}$ after hysterectomy. The conflicting terminology benign and metastasizing reflects its benign histological appearance in contrast to the presence of metastases from the primary site (uterus) to other organs, such as lungs, lymph nodes, bones, skeletal muscle, and the retroperitoneal space.

Several theories have been proposed for the pathogenesis of BML, including the presence of undetected lowgrade uterine leiomyosarcoma with metastases. ${ }^{2}$ The incidence of malignancy (eg, leiomyosarcoma) is low and ranges between $0.13 \%$ and $6 \% .{ }^{3}$ Patients with leiomyomas with a mitotic index of 5-9 per 10 high-power field or greater, cellular atypia, and coagulative tumor cell necrosis are associated with having malignant activity and should be managed as having leiomyosarcoma. ${ }^{4}$

A second theory is lung emboli of cells originating from a benign leiomyoma of the uterus. A unified histogenetic view of leiomyoma with vascular microinvasion, BML, and intravenous leiomyomatosis has been proposed. ${ }^{5}$ Some authors suggest that surgical manipulation may predispose to bloodstream dissemination. ${ }^{6}$

A third theory is systemic leiomyomatosis with multifocal (in various organs) but independent muscle proliferation, resulting from an abnormal sexual hormonal status. ${ }^{7}$

In our case, the more plausible theory seems to be that of intravascular seeding of benign smooth muscle cells during the initial operation. The absence of foci of cellular atypia in pulmonary nodules and residual uterine and peritoneal tissues excludes the presence of low-grade leiomyosarcoma. In addition, normal chest x-ray at the initial surgery, a prolonged ensuing time interval, and absence of extrathoracic lesions in the CT scan make the synchronous multifocal leiomyomatosis theory less likely.

The lung is the most common site of involvement, with a typically indolent clinical course. Typical radiographic presentation is well-circumscribed solitary or multiple pulmonary nodules with smooth or lobulated margins, unilaterally or mostly bilaterally distributed. Lesion size ranges from a few millimeters to several centimeters. ${ }^{8}$ The nodules do not display contrast enhancement in CT scans and rarely present calcification, cavitation,,${ }^{9,10}$ or cystic degeneration, ${ }^{11}$ frequently complicated by pneumothorax. The radiological imaging appearance of multiple pulmonary nodules with cavitation mimics many entities that have to be included in the differential diagnosis: metastases from malignant tumors such as sarcoma, squamous cell cancer (especially spindle cell subtype), transitional cell carcinoma of the bladder, melanoma and less frequently lymphoma, the multinodular form of bronchioalveolar cell carcinoma, anti-neutrophil cytoplasmic antibody-associated granulomatous vasculitis, necrotizing bacterial pneumonia or multifocal parenchymal infarctions, granulomas due to fungal or mycobacterial infections, and the rare smokingrelated Langerhans cell histiocytosis.

PET/CT scan findings in our subject were consistent with previously published cases reporting non-avid or mild 18F-FDG uptake (maximum standard uptake value of $<3$ ) by pulmonary nodules. ${ }^{12,13}$

The presence of estrogen and progesterone receptors has been documented in most cases of BML, and it is likely that the growth and progression of BML are closely related to stimulation by reproductive hormones. ${ }^{14}$ Since BML is a hormone-dependent tumor, the therapy objective is to prevent proliferation of smooth muscle cells due to hormonal stimulation by reducing estrogen levels. The choice of treatment can be difficult, especially for younger women of reproductive age. Bilateral salpingo-oophorectomy without estrogen replacement therapy is usually effective. ${ }^{15}$ Hormonal treatment (gonadotropin-releasing hormone analogues, progestins, tamoxifen, raloxifene, or aromatase inhibitors) is reported as an alternative method 
in select patients with beneficial effects, ${ }^{16}$ obviating the need for a surgical procedure or allowing symptom control when surgical management is not possible. Occasionally, pelvic surgery combined with hormonal treatment has been proposed. ${ }^{17}$ Other reported treatment methods include chemotherapy, ovarian radiotherapy, pulmonary nodule resection, and careful observation, especially in women in menopause with declining estrogen levels. Most BML lesions remain constant or decrease in size during follow-up after bilateral salpingo-oophorectomy. ${ }^{15}$ Long-term follow-up is recommended in all cases of BML because of the potential risk of recurrence ${ }^{4}$ and malignant behavior despite oophorectomy or hormonal suppression. PET/CT scan may reveal mild 18F-FDG uptake, and the potential for lowgrade malignancy cannot be excluded if the pulmonary nodule has not been biopsied and/or surgically removed. In our subject, the number, size, location and morphology of the pulmonary lesions remained stable in a CT scan performed $1 \mathrm{y}$ later.

BML, presenting as cavitating pulmonary nodules, is a rare clinical entity and should be part of the differential diagnosis of women with a history of hysterectomy.

\section{REFERENCES}

1. Steiner PE. Metastasizing fibroleiomyoma of the uterus: report of a case and review of the literature. Am J Pathol 1939;15(1):89-110.

2. Jautzke G, Müller-Ruchholtz E, Thalmann U. Immunohistological detection of estrogen and progesterone receptors in multiple and well differentiated leiomyomatous lung tumors in women with uterine leiomyomas (so-called benign metastasizing leiomyomas). A report on 5 cases. Pathol Res Pract 1996;192(3):215-223.

3. Robboy SJ, Bentley RC, Butnor K, Anderson MC. Pathology and pathophysiology of uterine smooth-muscle tumors. Environ Health Perspect 2000;108(Suppl 5):779-784.

4. Awonuga AO, Rotas M, Imudia AN, Choi C, Khulpateea N. Recurrent benign metastasizing leiomyoma after hysterectomy and bilateral salpingo-oophorectomy. Arch Gynecol Obstet 2008;278(4):373376.
5. Canzonieri V, D’Amore ES, Bartoloni G, Piazza M, Blandamura S, Carbone A. Leiomyomatosis with vascular invasion. A unified pathogenesis regarding leiomyoma with vascular microinvasion, benign metastasizing leiomyoma and intravenous leiomyomatosis. Virchows Arch 1994;425(5):541-545.

6. Egberts JH, Schafmayer C, Bauerschlag DO, Jänig U, Tepel J. Benign abdominal and pulmonary metastasizing leiomyoma of the uterus. Arch Gynecol Obstet 2006;274(5):319-322.

7. Cho KR, Woodruff JD, Epstein JI. Leiomyoma of the uterus with multiple extrauterine smooth muscle tumors: a case report suggesting multifocal origin. Hum Pathol 1989;20(1):80-83.

8. Abramson S, Gilkeson RC, Goldstein JD, Woodard PK, Eisenberg $\mathrm{R}$, Abramson N. Benign metastasizing leiomyoma: clinical, imaging, and pathologic correlation. AJR 2001;176(6):1409-1413.

9. Shin MS, Fulmer JD, Ho KJ. Unusual computed tomographic manifestations of benign metastasizing leiomyomas as cavitary nodular lesions or interstitial lung disease. Clin Imaging 1996;20(1):45-49.

10. Fasih N, Prasad Shanbhogue AK, Macdonald DB, Fraser-Hill MA, Papadatos D, Kielar AZ, et al. Leiomyomas beyond the uterus: unusual locations, rare manifestations. Radiographics 2008;28(7):19311948.

11. Gotti G, Haid MM, Paladini P, Di Bisceglie M, Volterrani L, Sforza V. Pedunculated pulmonary leiomyoma with large cyst formation. Ann Thorac Surg 1993;56(5):1178-1180.

12. Lin X, Fan W, Lang P, Hu Y, Zhang X, Sun X. Benign metastasizing leiomyoma identified using 18F-FDG PET/CT. Int J Gynaecol Obstet 2010;110(2):154-156.

13. di Scioscio V, Feraco P, Miglio L, Toni F, Malvi D, Pacilli AM, et al. Benign metastasizing leiomyoma of the lung: PET findings. J Thorac Imaging 2009;24(1):41-44.

14. Jiang GQ, Gao YN, Gao M, Zheng H, Yan X, Wang W, et al. Benign metastasizing leiomyoma: report of two cases and literature review. Chin Med J 2010;123(22):3367-3371.

15. Banner AS, Carrington CB, Emory WB, Kittle F, Leonard G, Ringus $\mathrm{J}$, et al. Efficacy of oophorectomy in lymphangioleiomyomatosis and benign metastasizing leiomyoma. N Engl J Med 1981;305(4):204209.

16. Rivera JA, Christopoulos S, Small D, Trifiro M. Hormonal manipulation of benign metastasizing leiomyomas: report of two cases and review of the literature. J Clin Endocrinol Metab 2004;89(7):31833188 .

17. Nasu K, Tsuno A, Takai N, Narahara H. A case of benign metastasizing leiomyoma treated by surgical castration followed by an aromatase inhibitor, anastrozole. Arch Gynecol Obstet 2009;279(2):255257. 